\title{
Luminescence from hydrodynamic cavitation
}

M. Farhat, A. Chakravarty and J. E. Field

Proc. R. Soc. A published online 30 June 2010 doi: 10.1098/rspa.2010.0134

\section{References}

$\mathbf{P}<\mathbf{P}$

\section{Subject collections}

\section{Email alerting service}

This article cites 15 articles

http://rspa.royalsocietypublishing.org/content/early/2010/06/24/rs pa.2010.0134.full.html\#ref-list-1

Published online 30 June 2010 in advance of the print journal.

Articles on similar topics can be found in the following collections fluid mechanics (127 articles)

Receive free email alerts when new articles cite this article - sign up in the box at the top right-hand corner of the article or click here

Advance online articles have been peer reviewed and accepted for publication but have not yet appeared in the paper journal (edited, typeset versions may be posted when available prior to final publication). Advance online articles are citable and establish publication priority; they are indexed by PubMed from initial publication. Citations to Advance online articles must include the digital object identifier (DOIs) and date of initial publication.

To subscribe to Proc. R. Soc. A go to: http://rspa.royalsocietypublishing.org/subscriptions 


\title{
PROCEEDINGS
}
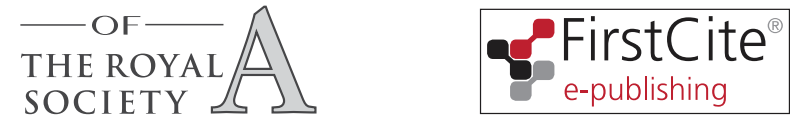

\section{Luminescence from hydrodynamic cavitation}

\author{
By M. Farhat ${ }^{1}$, A. Chakravarty ${ }^{2}$ and J. E. Field ${ }^{2, *}$ \\ ${ }^{1}$ Ecole Polytechnique Fédérale de Lausanne (EPFL), Laboratory of Hydraulic \\ Machines, Lausanne, Switzerland \\ ${ }^{2}$ Cavendish Laboratory, J J Thomson Avenue, Cambridge CB3 0HE, UK
}

The majority of the research on cavitation luminescence has focused on the sonoluminescence or chemiluminescence generated by cavitation induced through ultrasound, with a lesser body of work on the luminescence induced by laser- or sparkinduced cavitation. In such circumstances, the cavitation is generated in liquids where, on the broad scale, there is usually assumed to be no net liquid flow (although of course there are small-scale flows as a result of the cavitation itself, through radiation forces, streaming, microstreaming and turbulence). Little attention has been paid to the luminescence that accompanies (undesirable) cavitation in pumps and turbines or in marine propellers. In the present study, the sonoluminescence specific to air/water vapour bubbles, collapsing within a cavitation tunnel, is addressed. The particular case of leading edge cavitation over a two-dimensional hydrofoil is considered in detail. Hence, strong instabilities develop, causing the attached cavity to shed large clouds of micro bubbles. The spatial and temporal properties of the emitted luminescence were studied using an intensified charge coupled device video camera and a photomultiplier (PM). The light emission was found to extend downstream from the region of cavity closure, to the region where the travelling vortices collapse. Examination of the PM signal on short time scales showed that the emitted luminescence consisted of relatively intense flashes of short duration (as with other forms of luminescence). Individual flashes were often found to be clustered in time. Over longer time scales, clear evidence of periodicity was found in the PM signals. Further analysis showed that bursts of light were being emitted at the Strouhal frequency (for the shedding of transcient cavities).

Keywords: cavitation; luminescence; high-speed photography; bubble collapse; erosion; turbines

\section{Introduction}

Liquids, unlike gases, can withstand negative pressures, but if the tension is maintained for a sufficient time the liquid fails. Voids, or bubbles, are created within the bulk of the liquid; and these, unlike the liquid itself, cannot support any load. As such, the bubbles continue to grow until the tension in the liquid is relieved. If, at some point, the pressure in the liquid returns to a positive *Author for correspondence (jef1003@cam.ac.uk).

The paper is dedicated to Dr Chakravarty who tragically died while walking in the Grand Canyon, USA. 
value, an interesting situation results. Though the pressure in the liquid is restored, the pressure within the bubbles remains essentially zero, and so the bubbles must collapse. The most well-known analysis of this form of bubble collapse was given by Lord Rayleigh (1917). Rayleigh considered the idealized case where the rate at which the collapse of a (spherical) bubble proceeds was limited solely by the inertia of the liquid. What makes this model interesting, from the physical standpoint, is that the bubble wall accelerates without limit: the pressure in the liquid near the bubble wall and the velocity of the bubble wall become infinite at the end of the collapse. In practice, besides viscous forces, the presence of a finite mass of gas and uncondensed vapour within the bubble halts the collapse as the pressure within the bubble increases, leading to high gas temperatures and pressures at the end of the collapse, followed by bubble rebound. The extreme conditions which are predicted to occur in the gas are mirrored by the experimental observations that the collapse of cavitation bubbles is accompanied by chemical reactions and the emission of light. The light emission was first noted in the case of liquids cavitated by ultrasound (Frenzel \& Schultes 1934), and is generally termed sonoluminescence (SL). While models concentrated on the dynamics of single bubbles, usually with the assumption that they remain spherical at all times, experimental work was for decades dominated by studies of multibubble sonoluminescence (MBSL), the emission generated from clouds of bubbles. This is because the insonification conditions and applications tended to produce cavitation clouds. In recent years, an acoustic technique for producing repetitive single-bubble sonoluminescence (SBSL) has received considerable attention owing to the remarkable properties of the emitted light (Barberet al. 1997).

The motivation for Rayleigh's analysis was to aid the understanding of the cavitation erosion associated with hydrodynamic flow, a form of damage that was of interest to the designers of marine propellers. In this case, the pressure changes driving cavitation are not imposed externally, but arise from variations in the local velocity of the liquid within a non-uniform flow field (a consequence of the Bernoulli effect). An increase in the local velocity of a liquid causes the pressure within it to fall. If the velocity is sufficiently high, the liquid will be under tension and cavitation bubbles will grow naturally. These bubbles will eventually be convected into a region of lower velocity, where the higher pressure causes the bubbles to collapse. Rayleigh considered the simplest case of a cavity that remained spherical at all times; the extent to which this assumption was valid, in this context, remained unclear for some years.

Knapp \& Hollander (1948) investigated the cavitation induced by pressure drop over a blade using high-speed photography, and found the cavitation bubbles to be strongly deformed by the flow. Some bubbles were almost hemispherical in shape, being separated from the surface of the blade by a thin layer of liquid. These observations were confirmed by Ceccio \& Brennen (1991) and Guennoun et al. (2003) among many others. In view of this, the absence of luminescence, reported by Harvey (1939) for water cavitated by the action of a propeller, may seem unremarkable. Nevertheless, it was eventually discovered that the addition of a small quantity of xenon gas to the liquid being cavitated by flow, enabled the luminescence from the cavitation bubbles to be detected with ease (Peterson \& Anderson 1967). It would appear that the intensity of the SL from aspherical bubbles has a very strong dependence on the particular dissolved gases present. 
For example, the SL from xenon bubbles produced within water flowing through a Venturi tube (another instance of flow-induced cavitation) is 5000 times brighter than the SL from helium bubbles in the same situation; whereas in SBSL, where symmetry is maintained, the difference in intensity between the two gases is only a factor of five (Weninger et al. 1999).

Our poor level of understanding of the mechanism by which light is emitted in SL leaves considerable uncertainty as to why the SL from the heaviest noble gas bubbles is least affected by aspherical bubble collapse. Weninger et al. (1999) suggested that the trend in the intensity of the SL from the different noble gases may reflect any or all of the group trends; the thermal conductivity, sound speed and ionization potential all decrease down the group. In the (adiabatic) hot spot theory, a low thermal conductivity will lead to SL of greater intensity; in the shock wave model, a lower sound speed will enhance the converging shock; and in the plasma model a low ionization potential would lead to greater bremsstrahlung emission. These considerations are applicable to all forms of SL, irrespective of whether bubble symmetry is maintained. In the case of an aspherically collapsing bubble, however, any shocks launched from the bubble wall will not converge. For an aspherical collapse, therefore, it is probable that the ionization potential and thermal conductivity of the gas are important. Another significant feature of hydrodynamic cavitation is that the cavitation luminescence is typically associated with a single cycle of the growth and collapse of cavities, unlike acoustically induced SL, where the bubbles may continue to produce SL over many cycles. Nevertheless, Weninger et al. note that the intensities of the luminescence from bubbles of air, oxygen and deuterium collapsing in a Venturi tube are all at least 5000 times lower than the SL intensity of xenon bubbles (i.e. almost at the noise level of their measurements). This behaviour may be understood in the context of the adiabatic theory, where the adiabatic exponent, $\gamma$ (the ratio of the specific heats of the gas), is higher for monatomic gases than for more complicated molecules, which have vibrational and rotational degrees of freedom. A lower value of $\gamma$ will lead to a lower temperature for a given degree of compression. If sufficiently high temperatures are attained by diatomic (and polyatomic) gases, then the constituent molecules will dissociate; this occurs at around $9000 \mathrm{~K}$ for nitrogen and oxygen, and thus air, for example. The process of dissociation will absorb considerable quantities of energy, reducing the temperature attained on adiabatic compression. This energy will be released on the recombination of the molecular fragments, predominantly in the form of kinetic energy; very little of the energy will be released as light luminescence, as radiative recombination is a highly inefficient process for most ordinary diatomic gases. Only when the gas reaches temperatures at which an appreciable quantity of ionization could take place would significant light emission be expected. Of course, it should be emphasized that this reasoning is largely speculative; most experimental and theoretical work, in recent times, has focused on SBSL from noble gas bubbles. The characteristics of the SL from diatomic gases (other than hydrogen) have not been studied in any detail, because of difficulties in preventing these gases from engaging in sonochemical reactions. As has been noted, this problem is avoided in studies of luminescence from hydrodynamic cavitation. The present paper describes the results of an investigation into the luminescence from the hydrodynamic cavitation of water, containing dissolved air. 


\section{Cavitation tunnel—earlier studies}

The form of luminescence examined in this study was produced by the flow over a hydrofoil within a cavitation tunnel. This study builds on earlier work (Leighton et al. 2003) in which cavitation luminescence was studied via a photon-counting technique. The motivation for these studies is not limited to addressing questions about the physics of luminescence: hydrodynamic cavitation remains a significant engineering problem in high-speed hydraulic machinery. Not only is cavitation associated with erosion, but also it causes noise and vibrations, and may affect the efficiency of a hydraulic system, such as a pump or a turbine. The level of understanding of hydrodynamic cavitation is not sufficiently advanced to permit its occurrence and effects to be predicted computationally, and hence it continues to be necessary nowadays to test experimental models to determine the merits (or otherwise) of a given hydraulic design. Many diagnostic techniques have been employed to study this form of cavitation: photography, hydrophones, wall pressure and accelerometers, light scattering, surface pitting and so on. All of these techniques have advantages and disadvantages; given the strongly inhomogeneous nature of the cavitation caused by flow over a hydrofoil, it is often desirable to obtain a high degree of spatial resolution. This is most directly provided by the use of photography; however, if cavitation damage is of primary interest, the microscopically small size of the bubbles at the point of collapse can cause difficulties. Imaging the luminescence from the collapsed bubbles provides a means by which these problems may be overcome.

van der Meulen (1986) obtained photographs of the SL associated with cavitation on a hydrofoil. In his studies, the water in the cavitation tunnel was degassed and doped with xenon, typically to a concentration of $18 \mathrm{mll}^{-1}$. This value is similar to the concentration of air in water, saturated at atmospheric pressure. However, the solubility of xenon in water $\left(240 \mathrm{mll}^{-1}\right.$, atm) is much higher than that of air, hence the behaviour of the solution was similar to that of degassed water (the trend in the solubility of gases has an inverse correlation with the 'volatility' of the gas). By varying the hydrofoil incidence angle, van der Meulen was able to identify four distinct regimes of cavitation behaviour. At the shallowest angles $\left(2^{\circ}\right)$, the cavitation bubbles were of hemispherical form and remained separate from one another. As the angle of attack was increased to approximately $4^{\circ}$, separated bubbles gave way to a continuous cavity, which took the form of a sheet attached to the leading edge of the foil. When the angle of attack was increased further $\left(7^{\circ}\right)$, the sheet became progressively more unstable, giving rise to the production of clouds of cavitation bubbles. At the highest angle of attack $\left(12^{\circ}\right)$, the boundary layer separates at the leading edge of the hydrofoil and the cavities form in the core of the vortical structures generated in the recirculation zone.

Other than at the lowest angle of attack, he found that the intensity of the emitted cavitation luminescence increased with increasing velocity of the water flow (as the water velocity is perturbed by the hydrofoil, a reference velocity, $C$, upstream of the blade is generally quoted). He interpreted the results in terms of power-law behaviour, the exponent of $C$ being between 3.9 and 7.2. Similarly, he found the noise (measured using a hydrophone) generated by the cavitation was also positively correlated to $C$, with an exponent between 4.8 
and 5.9. Measurements of erosion rate (determined by counting pits on a copper hydrofoil) indicated that it was approximately proportional to the intensity of the luminescence, except at the lowest angles of attack.

van der Meulen suggested that the measures of the luminescence, noise and erosion should all be correlated if they each referred to the same volume of cavitation. However, as the erosion data were a measure of the cavitation occurring within a small distance of the surface of the foil, and the noise data corresponded to all cavitation, irrespective of where it occurred, the identification of the power-law scaling of the various measures of cavitation activity was thought to be of more significance than direct comparisons. An anomaly was noted in the case of bubble cavitation (i.e. at the lowest angles of attack): the intensity of the SL declined for increasing $C$, this effect appearing to follow the dramatic decline in the number of cavitation bubbles with increasing velocity. As the (hemispherical) bubbles remain at the surface of the foil, bubble cavitation was found to be associated with high pitting rates. Similarly, the luminescence from hemispherical xenon bubbles has been produced acoustically (Weninger et al. 1997). Van der Meulen did not study the temporal properties of the luminescence from hydrodynamic cavitation beyond the visual observation that 'bright flashes could be observed occasionally, in addition to the more or less continuous light emission'.

The first attempt to obtain information on the absolute intensity, and temporal properties of the cavitation luminescence from flow over a hydrofoil was made by Leighton et al. (2003), who carried out studies in the EPFL high-speed cavitation tunnel (Avellan et al. 1987). The test section of the tunnel is $150 \times 150 \mathrm{~mm}$ and $750 \mathrm{~mm}$ long, with a maximum water velocity of $50 \mathrm{~m} \mathrm{~s}^{-1}$. To cover the range of possible signals that might occur, given the lack of a priori information, Leighton et al. used a photomultiplier (PM) in conjunction with a pulse counter. The signal from the pulse counter was passed through a dual-level discriminator (set with a dynamic range within $6 \mathrm{~dB}$ per bit specifically so that the clipping and digitization errors would not occur). Each photon pulse was increased to a standard image intensifier tube pulse of $1 \mu$ s duration. If it is assumed as a working model that two luminescent emissions separated by greater than $1 \mu \mathrm{s}$ correspond to two separate bubble collapses, then the photon count in the Leighton et al. (2003) experiment represents the number of cavitation events. Long records were taken because of the possibilities of information content at the shedding frequencies (of the order of tens of hertz). Since the available acquisition system could not store so much data, instead the counts were binned into time windows of $20 \mathrm{~ms}$ durations, with a $200 \mu \mathrm{s}$ dead-time between each window, and the system stored the statistics of how many of these windows contained what number of cavitation events. The effect of this discretization, and the possibility and effect of undercounting, were quantitatively addressed. Data were obtained for various flow parameters, with the upstream velocity $C$ ranging from less than 18 to $32 \mathrm{~m} \mathrm{~s}^{-1}$, and the cavitation number, $\sigma$ ranging from less than 0.9 to 1.5. Here, the cavitation number is defined as follows: $\sigma=2\left(p_{\text {ref }}-p_{\mathrm{v}}\right) / \rho C^{2}$, where $p_{\text {ref }}$ is the pressure at the inlet to the test section, $p_{\mathrm{v}}$ the vapour pressure and $\rho$ the liquid density. The highest rate of cavitation events was estimated to be around $3 \times 10^{7} \mathrm{~s}^{-1}$. For a NACA 009 (figure 1) hydrofoil $150 \mathrm{~mm}$ wide, truncated at $100 \mathrm{~mm}$ (90\% chord length), and an angle of attack of $4^{\circ}$, it was found that both cavitation and luminescence emissions were 


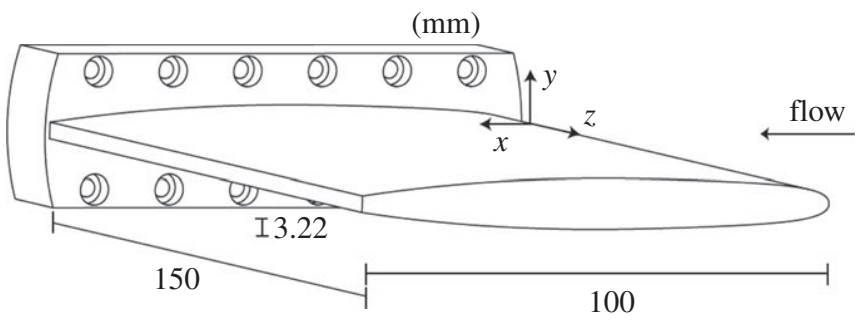

Figure 1. NACA 009 hydrofoil.

detected for velocities greater than $17 \mathrm{~ms}^{-1}$. The number of photons detected increased with increasing $C$, but with a decrease in the rate of increase at higher velocities (approx. $30 \mathrm{~m} \mathrm{~s}^{-1}$ ). Three possible explanations, pertaining to what happens at higher flow speeds, were given for this: (i) owing to a possible Reynolds effect as already reported, the source of luminescence might extend beyond the field of view of the PM, (ii) the non-luminescing bubble cloud extends to obscure the luminescent bubbles, and (iii) that the ratio of collapses that generate luminescence, compared with the total number of collapses, decreases with increasing flow velocity (i.e. it is 1 in 17 for $C=32.1 \mathrm{~m} \mathrm{~s}^{-1}$, whereas it was 1 in 4 for $C=18.3 \mathrm{~m} \mathrm{~s}^{-1}$ ). The data on the occupancy of the windows were found to depart from the Poisson distribution, indicating that the light was emitted in bursts. Limits were estimated for the probable frequencies associated with these bursts, and preliminary tests undertaken to compare these with the shedding frequency using the Strouhal number (estimated to take a value of approx. 0.3). To distinguish between options (i)-(iii), to estimate the burst frequencies to greater accuracy and to more fully exploit the Strouhal number, $S=f l_{\mathrm{c}} / C$, where $f$ denotes the shedding rate of the transient cavities, $l_{\mathrm{c}}$ the main cavity length, and $C$ the upstream velocity. Leighton et al. suggest that 'In future research, an image intensifier system could be used to give spatial resolution similar to the photographic work of van der Meulen \& Nakashim (1983), but with video time resolution. Now that the upper limit on the interburst rate is known, a system could be designed which exploits PM current to provide a time series suitable for rate analysis. This would allow more detailed research on the shedding and transient collapse processes'. This paper undertakes that study.

\section{Cavitation tunnel-new research}

It was decided, in the present investigation, to obtain further measurements of the system studied by Leighton et al. with a PM and an image intensifier. In the case of the PM measurements, direct use of the anode signal was made (i.e. without the use of pulse counting electronics). The PM used was of circular focused construction (EMI type 9781B, bialkali photocathode, 200-650 nm response, quantum efficiency $20 \%$ at $400 \mathrm{~nm}$, rise time $2 \mathrm{~ns}$ ). Its output was recorded by an oscilloscope (LeCroy LC574) having an analogue bandwidth of $1 \mathrm{GHz}$, and a maximum sampling rate of $2 \mathrm{GS} \mathrm{s}^{-1}$. Imaging of the region responsible for the emission of luminescence was achieved with the aid of an intensified charge coupled device (CCD) video camera (4Quik05) operated at maximum 
gain (approx. 40 000), which was sufficient to ensure that single photons could be recorded. The intensifier had an S-20 photocathode (300-820 nm, peak quantum efficiency $20 \%$ at $420 \mathrm{~nm}$ ). The camera was equipped with a lens of large aperture ratio $(f / 1.8)$, in order to collect as much light as possible; and the output of the camera was recorded using frame grabber software (Matrox Intellicam). Generally, the camera was used in the non-interlaced mode, giving 50 frames per second (19.5 ms exposure) of $290 \times 750$ pixels on a greyscale of 255 levels; the 'dark' noise in the CCD extended up to level 30 and single photons were typically recorded at level 40 or above. The frames were either recorded in sequence, or several frames were automatically summed, the noise level in the CCD being automatically subtracted. Both the camera and the PM were set to view the foil from above, through a window that did not transmit UV radiation.

The velocity of the water at the inlet to the test section was set within the range $15-35 \mathrm{~m} \mathrm{~s}^{-1}$, with the hydrostatic pressure adjusted to maintain a constant cavitation number $(\sigma=0.95)$; the angle of attack of the hydrofoil, $\alpha$, was fixed at $3.5^{\circ}$. These conditions led to the formation of an attached cavity, which extends across the width of the foil and occupies some 40 per cent of its chord-wise length (Couty et al. 2001). Thanks to a specific bubble trap system, all the tests are carried out with well-degassed water. The dissolved oxygen concentration is maintained below $3 \mathrm{ppm}$ with less than one active nuclei per cubic centimetre.

The shedding process of the travelling vortices is closely related to the dynamic behaviour of the attached cavity. The so-called 'sheet cavitation' is characterized by a random and low-amplitude oscillation of the main cavity. In this case, the size of transient cavities is small compared with the cavity length. As the turbulence level is increased, whether by increasing the flow velocity or the incidence angle, a transition to the so-called 'cloud cavitation' takes place. In this case, the shedding process is controlled by a re-entrant jet, which leads to a periodic shedding of large transient cavities governed by a Strouhal-type law (Farhat et al. 1993). We have presented in figure 2 flash illuminated exposures of the cavitation behaviour observed from the top window for 20, 26 and $30 \mathrm{~m} \mathrm{~s}^{-1}$ upstream velocity. For flow velocity of $20 \mathrm{~m} \mathrm{~s}^{-1}$, a sheet cavitation is observed with low amplitude fluctuation and a random shedding of transient cavities. As the velocity is increased beyond $20 \mathrm{~m} \mathrm{~s}^{-1}$, strong instabilities appear with a substantial increase of the size of transient cavities. In our case, the Strouhal number, estimated with a stroboscopic light, lies between 0.25 and 0.28 approximately for flow velocities above $20 \mathrm{~m} \mathrm{~s}^{-1}$. We have presented in the same figure, exposures of the emitted SL taken using the intensified CCD camera at similar flow velocities. Each of the SL photographs consists of 50 consecutive frames of video that have been summed to give an effective exposure of $1 \mathrm{~s}$. This exposure time is significantly shorter than that used by van der Meulen (30 min). Accordingly, it is still possible to distinguish individual SL events as spots of light in figure 2: the luminescence appears in the form of small spots of varying brightness, the number and brightness of these spots increasing at the higher water velocities. In spatial terms, the SL emission is essentially confined to the region downstream of the trailing edge of the attached cavity, extending beyond the trailing edge of the foil (particularly at high water velocity, where the larger size and velocity of the cavities shed enable them to travel greater distances before collapsing). One may easily observe that the density of SL sources is significantly higher at the upper part of the 

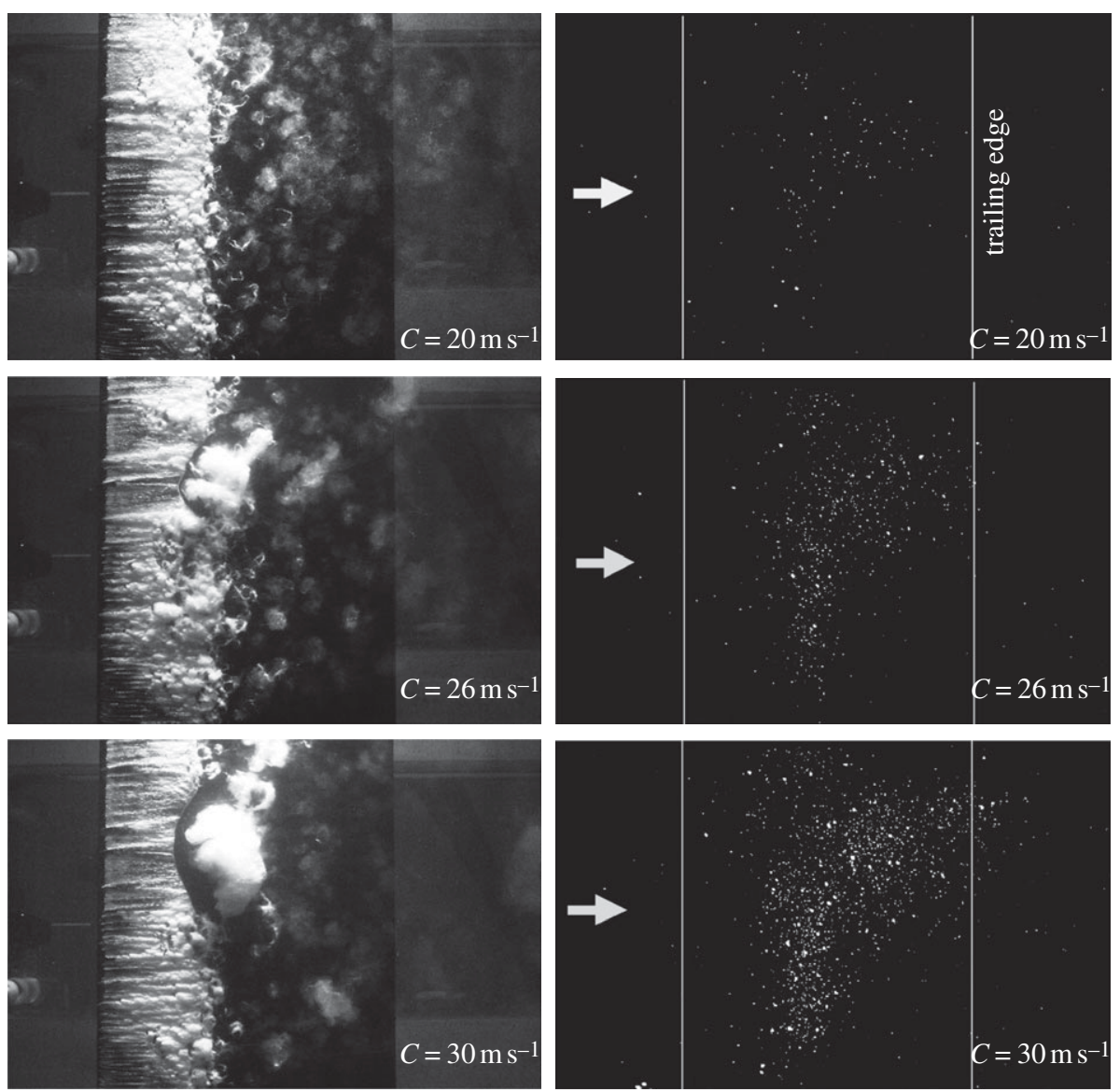

Figure 2. Flash-illuminated photographs of the leading edge cavitation with their corresponding $1 \mathrm{~s}$ exposures of the SL, at 20,26 and $30 \mathrm{~m} \mathrm{~s}^{-1}$ upstream velocities.

photograph. This correlates remarkably well with the fact that large cavities are shed in this same area of the hydrofoil as shown by photographs presented in the same figure.

Measurements with the PM confirmed that the light emission consisted of multi-photon flashes of very short duration. The measured width of the PM pulses ranged from 1.8-6.5 ns, the average figure being around $2.4 \mathrm{~ns}$ (figure 3 ), this corresponds to an effective SL flashwidth of about 1 ns (having corrected for the instrument response). The intensity of the flashes varied over some orders of magnitude (figure 4), the frequency with which pulses of a given intensity were recorded declined dramatically with increasing intensity. The form of the pulse height distribution is similar to that measured by Peterson \& Anderson (1967) for SL produced within a Venturi tube. On examining the PM signals over longer time scales, records (such as those illustrated in figure 5) were obtained. The appropriate level of PM signal integration (approx. $300 \mu \mathrm{s}$ ) was achieved by switching from a $50 \Omega$ termination to a $1 \mathrm{M} \Omega$ termination on the oscilloscope. The bursts first visually identified by van der Meulen \& Nakashim (1983), and to which 


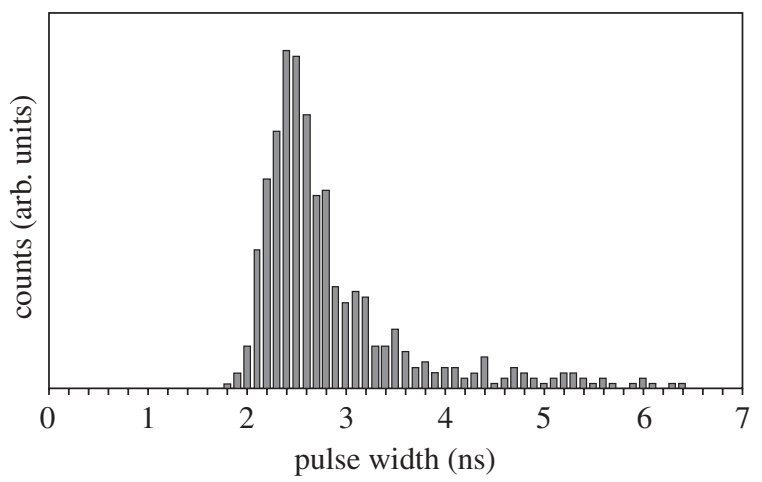

Figure 3. Histogram of the PM pulse rise time (20-80\%) in response to SL pulses $\left(C=30 \mathrm{~m} \mathrm{~s}^{-1}\right)$.

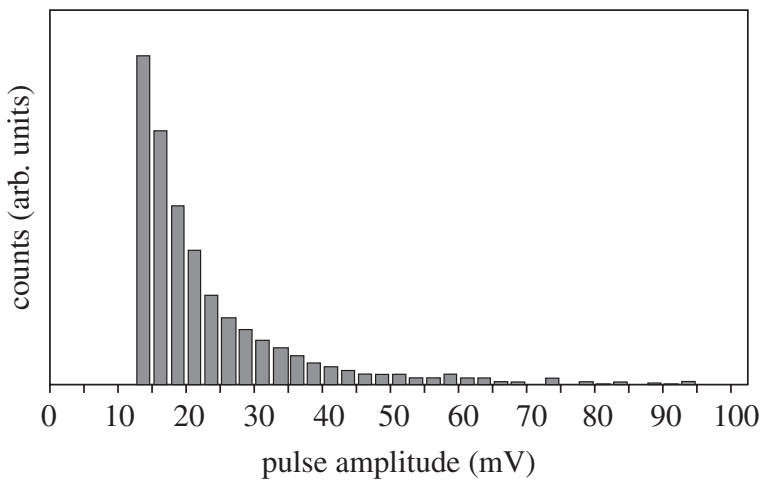

Figure 4. SL pulse height spectrum $\left(C=30 \mathrm{~m} \mathrm{~s}^{-1}\right)$. Only pulses greater than the oscilloscope trigger level $(12 \mathrm{mV})$ are recorded.

Leighton et al. (2003) assigned frequency limits that they attempted to identify with the shedding frequencies, have here been confirmed. The periodicities estimated here are in the range $100-250 \mathrm{~Hz}$, identified by inputting the PM signal into a Fourier transform algorithm. The results are presented in figure 6 , in which the frequency spectrum of the PM signals is expressed in a nondimensional form, $f^{*}=f l_{c} / C$, where $f$ is the frequency and $l_{c}$ the length of the attached cavity. The clear peak at $f^{*}=0.27$, independent of water velocity, shows that the periodicity of the luminescence signal matches the shedding frequency of the cavitation vortices from the attached cavity.

While the Strouhal numbers used in both studies agree, the estimated periodicity of $100-250 \mathrm{~Hz}$ is greater than the frequencies estimated by Leighton et al. (who placed an upper limit at approx. 20-40 Hz), and while van der Meulen \& Nakashim (1983) give no quantified estimates, their visual reports suggest that lower frequencies must have been present than $100-250 \mathrm{~Hz}$ (which would have appeared as continuous to the eye). For $C=15 \mathrm{~m} \mathrm{~s}^{-1}$ they say 'bright flashes could be observed occasionally, in addition to the more or less continuous light emission' (for a $3 \mathrm{~cm}$ cavity length and $\alpha=7^{\circ}$, they note that 


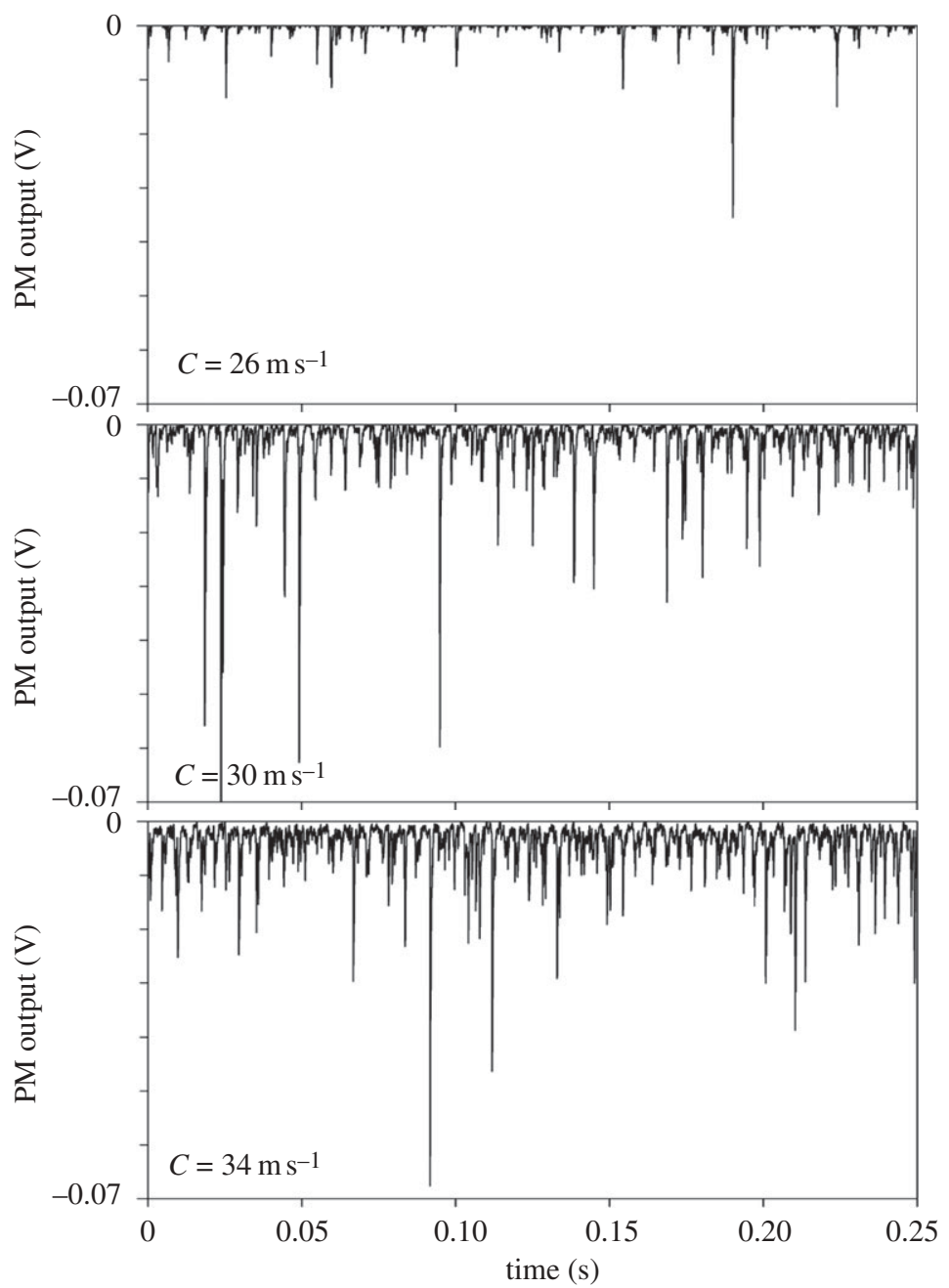

Figure 5. Temporal records of SL emission at different upstream velocities.

while luminescence was bright and constant from $25 \%$ to $55 \%$ of the way along the chord, from $50 \%$ to $90 \%$ along the chord 'bright flashes could be observed frequently' (van der Meulen \& Nakashim 1983).

The PM was also used to measure the average intensity of the luminescence signal. This was achieved by determining the average anode current as a function of upstream velocity and scaling this for the gain of the PM, the fraction of photons intercepted by the photocathode of the PM (1/2100, assuming isotropic emission), and the mean quantum efficiency of the photocathode for the spectrum of the emitted SL light. This last quantity was taken to be of the order 15 per cent based on an assumption of a substantially flat spectrum (no attempt was made to determine the true spectral behaviour). About $10^{10}$ photons are emitted per second (in the range $400-600 \mathrm{~nm}$ ) for a velocity of $34 \mathrm{~m} \mathrm{~s}^{-1}$. The intensity of the luminescence as a function of velocity is illustrated in figure 7 . 


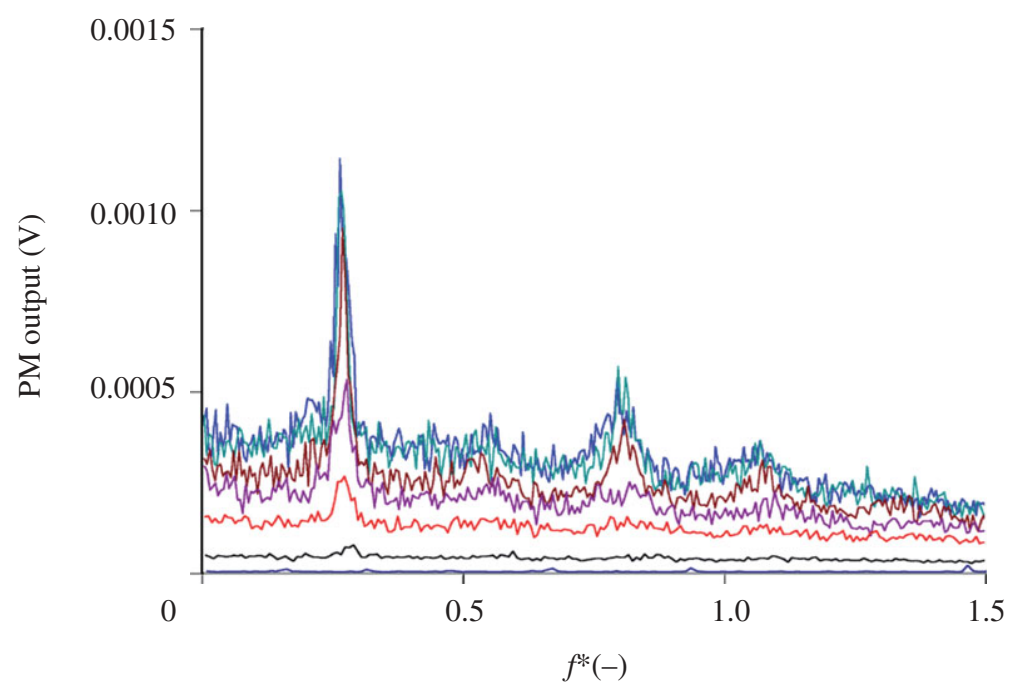

Figure 6. Frequency spectrum of the PM signals, expressed in terms of the non-dimensional frequency (blue, $C=34 \mathrm{~m} \mathrm{~s}^{-1}$; green, $C=32 \mathrm{~m} \mathrm{~s}^{-1}$; brown, $C=30 \mathrm{~m} \mathrm{~s}^{-1}$; magenta, $C=28 \mathrm{~m} \mathrm{~s}^{-1}$; red, $C=24 \mathrm{~m} \mathrm{~s}^{-1}$; black, $C=20 \mathrm{~m} \mathrm{~s}^{-1}$; violet, $C=15 \mathrm{~m} \mathrm{~s}^{-1}$ ).

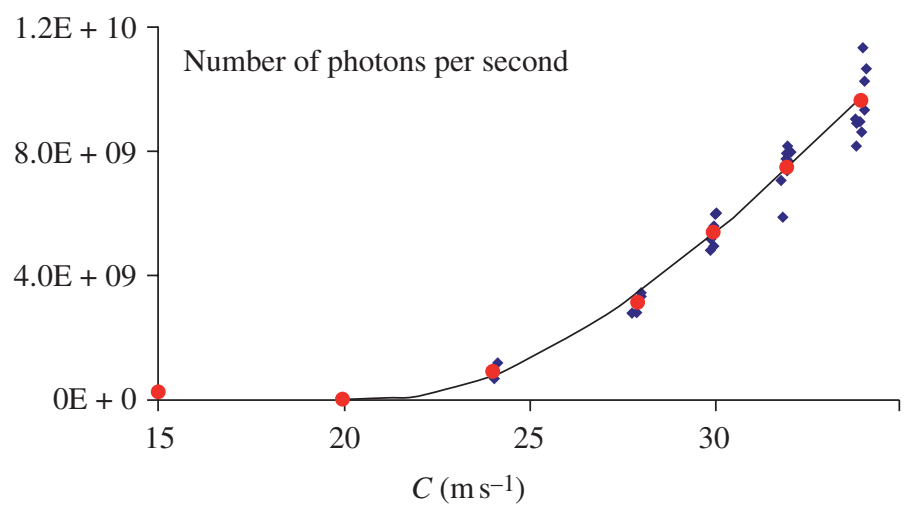

Figure 7. Mean intensity of the SL emission as a function of the upstream velocity (filled blue diamonds, photons arrival frequency; filled red circles, averaged values; solid line, cubic polynomial fitting).

The onset of the SL occurred at an upstream velocity of about $17 \mathrm{~m} \mathrm{~s}^{-1}$, in agreement with Leighton et al. However, the correct value of the onset velocity is not seen in figure 7, as the PM data were obtained by integration of digitally sampled temporal records (rather than by the direct analogue measurements). It is interesting to compare figure 7 of the current study (which records the number of luminescence photons detected) with fig. 9 of Leighton et al. (2003) (which records the number of cavitation events, within the limitations discussed in $\S 2$ ). The ratio of the two counts, for identical flow conditions, gives an estimation of the number of photons emitted by each event. This first-order value of this 
ratio, of up to approximately 300, should be treated as suggestive only given (i) the limitations discussed in $\S 2$ on interpreting the 2003 count in terms of cavitation events; (ii) the assumptions inherent in calculating the total photon emission in the current study (e.g. no multiple scattering of photons), and (iii) the fact that the flow conditions are not identical (in the current study $\sigma=0.95$ and $\alpha=3.5^{\circ}$, whereas in fig. 9 of Leighton et al. (2003) the values of $\sigma=1.1$ and $\alpha=4^{\circ}$ ). However, while the absolute value of the ratio can be seen only as preliminary estimate, the trend in the ratio is illuminating. In figure 7 the rate at which the photon count increases with increasing flow velocity shows no sign of the reduction seen in the number of cavitation events above $30 \mathrm{~m} \mathrm{~s}^{-1}$ in fig. 9 of Leighton et al. This increase in the ratio would suggest the concentration of the emission into fewer, brighter cavitation events, in support of option (iii) discussed in $\S 2$. This concentration into fewer more energetic collapses, when the conditions driving cavitation at a hydrofoil become more extreme, is well known to submariners through the 'anomalous depth effect'. Propeller cavitation in a submarine is extremely unwanted, as it can readily reveal the location of the vessel to passive sonar. Submariners know that, once a propeller has begun to generate cavitation, submerging will tend to reduce cavitation noise. However, when the cavitation is strong and the vessel is at high speed, increasing the depth of the vessel will first cause an increase in the cavitation noise, before suppression occurs. This so-called 'anomalous depth effect' is due to the fact that an increase in static pressure increases the violence of those individual collapses that do occur, while reducing the number of energetic cavitation events (Leighton 1998).

The evidence above in support of option (iii) is bolstered by the imageintensifier images (figure 2). These indicate that the spatial distribution of the luminescence (in the case addressed in the present work) is substantially independent of the velocity of the water flow, the effect of increasing velocity being to increase the average intensity of the luminescence. If options (i) and (ii) of $\$ 2$ were applicable, then such constancy with varying flow speed would not be expected. Hence the evidence is strongly in favour of option (iii).

Another explanation for the occurrence of very bright collapses may be found in the dynamics of the growth and collapse of bubbles that luminesce. As the water flows over the hydrofoil, the pressure drop causes the expansion of the microscopic bubble nuclei that existed in the flow prior to the test section. Inertial cavitation comprises explosive bubble growth followed by a sufficiently rapid collapse, and both stages must be present to generate luminescence. If the microscopic bubble nucleus is too small, then surface tension forces prevent the initial sudden growth, and inertial cavitation does not occur (Leighton 1998). This is because the Laplace pressure varies inversely with the bubble radius, and therefore increases rapidly with decreasing equilibrium bubble radius $\left(R_{0}\right)$, and is very great in the smallest nuclei (Leighton 1994).

Conversely, if the equilibrium radius $R_{0}$ of the bubble nucleus is initially too large, then it may grow, but insufficiently to then concentrate the energy sufficiently on collapse to generate luminescence. The time scales on which such large bubbles respond to pressure (i.e. grow during a rarefaction) are relatively slow compared with smaller bubbles (as evidenced by the approximately inverse relationship between bubble radius and natural frequency). Following Leighton (2007), it is possible to explain the energetics of the collapse through consideration of these time scales as follows. Holland \& Apfel (1989) considered the response 
of bubbles to a single cycle sound wave (starting with a tension half cycle) of acoustic pressure amplitude $P_{\mathrm{A}}$ and circular frequency $\omega$. This scenario is easily extrapolated for flow over a hydrofoil, since where the interval $\pi / \omega$ corresponds to the characteristic time taken for the bubble to convect through the reduced pressure at the hydrofoil. The pressure drop excites the bubble to grow, but there is a delay time in bubble response to this pressure drop, which is characterized as being the summation of three components, corresponding to contributions caused by surface tension $\left(\Delta t_{\sigma}\right)$, inertia $\left(\Delta t_{I}\right)$ and viscosity $\left(\Delta t_{\eta}\right)$, their sum being

$$
\Delta t_{\sigma}+\Delta t_{\mathrm{I}}+\Delta t_{\eta} \approx \frac{2 \sigma}{P_{\mathrm{A}}-P_{\mathrm{B}}} \sqrt{\frac{3 \rho_{0}}{2\left(P_{\mathrm{A}}-P_{\mathrm{B}}\right)}}+\frac{2 R_{0}}{3} \sqrt{\frac{\rho_{0}}{\Delta P_{\text {wall }}}}+\frac{4 \eta}{\Delta P_{\text {wall }}},
$$

where $\rho_{0}$ is the liquid density at equilibrium, $\eta$ the shear viscosity of the liquid, and where $P_{\mathrm{B}}$ the Blake threshold pressure (Leighton 1994), the degree of tension that must be generated in the liquid to overcome surface tension

$$
P_{\mathrm{B}} \approx p_{0}+\frac{8 \sigma}{9} \sqrt{\frac{3 \sigma}{2 R_{0}^{3}\left(p_{0}+2 \sigma / R_{0}\right)}},
$$

and where $\Delta P_{\text {wall }}$ is the time-averaged pressure difference across the bubble wall:

$$
\Delta P_{\text {wall }} \approx \frac{\left(P_{A}+P_{B}-2 p_{0}+\sqrt{\left(P_{A}-p_{0}\right)\left(P_{A}-P_{B}\right)}\right)}{3} .
$$

As stated above, bubbles that are too small to grow do not nucleate inertial cavitation. Of those which do grow, some will generate luminescence, and some will not. Consider the large-bubble limit of the range of bubble radii which can nucleate inertial cavitation. Here the issue is not with $P_{\mathrm{B}}$, and hence the dependence in equation (3.1) of the time for growth on initial bubble radius is primarily through the inertial term $\Delta t_{\mathrm{I}} \approx\left(2 R_{0} / 3\right) \sqrt{\rho / \Delta P_{\text {wall }}}$, which is proportional to $R_{0}$ (Leighton 2007). Therefore the larger the bubble, the more slowly it grows, and so during a given rarefaction cycle, the less the degree of growth it achieves. The maximum radius $R_{\max }$ achieved by a bubble during the growth phase of inertial cavitation is

$$
R_{\max } \approx \frac{4}{3 \omega}\left(P-p_{0}\right) \sqrt{\frac{2}{\rho_{0} P_{\mathrm{A}}}}\left(1+\frac{2\left(P_{\mathrm{A}}-p_{0}\right)}{3 p_{0}}\right)^{1 / 3}
$$

(Apfel 1981; Leighton 1994), which is independent of the initial bubble radius $R_{0}$. If the Rayleigh model of an empty cavity were to be used, then it would incorrectly predict that the larger the value of $R_{\max }$, the more probable is the bubble to emit bright luminescence.

Rayleigh (1917) considered the collapse of an empty cavity which remains spherical at all times, located in an incompressible liquid. The empty cavity initially has radius $R_{\max }$ and the wall is initially stationary, but then accelerates inwards under the external pressure in the liquid $p_{\text {ext. }}$. Since the cavity contains no gas, the liquid pressure $p_{\mathrm{L}}$ just outside the cavity is zero (if surface tension is assumed to be negligible). Thus the work done by the liquid pressure $p_{\text {ext }}$ far from the bubble, from that time until the cavity has contracted to a radius $R$ is given by 
$4 \pi p_{\text {ext }}\left(R_{\max }^{3}-R^{3}\right) / 3$. Rayleigh equated this to the kinetic energy acquired by the liquid. $\varphi_{\mathrm{KE}}=\frac{1}{2} \rho_{0} \int_{r=R}^{r=\infty} 4 \pi r^{2} \dot{r}^{2} \mathrm{~d} r=2 \pi \rho_{0} R^{3} \dot{R}^{2}$. This would imply that the larger $R_{\max }$, the brighter one might expect the luminescence to be. The reason why this is not so is because of the presence of gas and vapour within the bubble, giving it a finite internal pressure of $p_{\mathrm{i}}$, which in turn imparts a finite pressure in the liquid at the bubble wall, obtained by dynamically matching normal stresses across at the bubble wall (Leighton 1994):

$$
\begin{aligned}
p_{\mathrm{L}} & =p_{\mathrm{i}}-\frac{2 \sigma}{R}-\frac{4 \eta \dot{R}}{R}+0\left(\frac{\dot{R}}{c}\right) \\
& \Rightarrow p_{\mathrm{L}}=\left(p_{0}+\frac{2 \sigma}{R_{0}}-p_{\mathrm{v}}\right)\left(\frac{R_{0}}{R}\right)^{3 K}+p_{\mathrm{v}}-\frac{2 \sigma}{R}-\frac{4 \eta \dot{R}}{R}+O\left(\frac{\dot{R}}{c}\right) .
\end{aligned}
$$

This must be added to the energy balance used by Rayleigh as follows:

$$
\int_{R_{\max }}^{R}\left(p_{L}-p_{\text {ext }}\right) 4 \pi R^{2} \mathrm{~d} R=2 \pi \rho_{0} R^{3} \dot{R}^{2} .
$$

If the initial bubble nucleus is insufficiently small, then its contribution to $p_{\text {i }}$ when it has expanded to maximum radius $R_{\max }$ is sufficiently large to reduce the energetics of the subsequent bubble collapse to a level that will not generate luminescence. If the bubble nucleus is too small, it will not undergo the required degree of growth. In the intermediate range, the expansion ratio $R_{\max } / R_{0}$ will be critical, and as this increases the potential to generate bright luminescence should increase. The photographic evidence shows bubbles attaining centimetrescale sizes at maximum growth. If these were achieved while maintaining low $p_{i}$ (i.e. the initial bubble must not be too large, or the degree of bubble coalescence during growth must not be too great) then there is potential for very bright cavitation luminescence, such as could be seen by the unaided eye.

\section{Discussion and conclusions}

Measurements have been taken of the cavitation luminescence from air bubbles, and these have revealed essential similarities with the other known forms of luminescence (e.g. the short duration of the light flashes). Over longer time scales, the luminescence signal reveals clear properties, such as the Strouhal periodicity, which reflect global hydrodynamic behaviour.

The intensified images obtained (figure 2) clearly indicate that the spatial distribution of the SL emission (in the case addressed in the present work) is substantially independent of the velocity of the water flow, the effect of increasing velocity being to increase the average intensity of the luminescence. Thus, the plateau in luminescence intensity observed by Leighton et al. (2003) cannot be explained by a spatial shift of the region of emission out of the field of view of the detector. It is more probable that the use of a photon-counting detector, in the earlier work, resulted in a substantial underestimate of the numbers of photons emitted at the higher velocities. 
Determining how measurements of luminescence relate to cavitation erosion is more difficult, as erosion is caused by those bubbles that collapse in close proximity to the foil surface. Though the emission of luminescence unambiguously locates the point of bubble collapse in both spatial and temporal terms, it is difficult to determine the distance from the point of collapse to the foil surface using conventional imaging techniques. The dynamics of the bubbles are significantly modified when collapse takes place near a solid surface, often causing the bubble wall to involute and direct a liquid jet at the solid. Such jets are regarded as the major cause of the erosive action of bubbles; however, the asymmetry implied by jet formation leads to a dramatic reduction in the focusing of energy within the bubble. Accordingly, it is found that bubbles collapsing near a solid surface emit far less luminescence than isolated bubbles (Ohl et al. 1998, 1999). The low luminescence intensity of the bubbles responsible for erosion, the difficulty of estimating their distance from the hydrofoil and the scattering caused by the presence of other bubbles, limit the degree to which it is possible to correlate cavitation luminescence emission and erosion. The temporal properties of the detected luminescence signal are, by contrast, substantially insensitive to scattering media, the velocity of light being sufficiently great to prevent any ambiguity in timing. It is possible, therefore, that the greatest utility of luminescence measurements, to the understanding of hydrodynamic cavitation phenomena, may lie in temporal measurements. The multi-photon flashes of light, associated with cavitation luminescence, are easily distinguished from other light sources of low intensity. This feature may permit the measurement of cavitation luminescence in hydraulic machines outside the laboratory, where it may not be possible to ensure complete darkness. The complexity of hydrodynamic cavitation prevents detailed investigations into the properties of individual cavitation events; nevertheless, it does provide a route to producing luminescence from bubbles of any gas on a single-shot basis.

This research was performed at EPFL during visits by Dr Chakravarty and Professor Field. M.F. would like to thank the Swiss National Science Foundation for its support (project no 2100-063842.00/1) and Mr F. Guennoune for his valuable help. A.C.'s research was supported by the EPSRC on Contract GR/N65424/01. J.E.F. thanks the Royal Society of London, the Swiss National Science Foundation and EPFL for support of study leave visits to EPFL. Prof. F. Avellan is thanked for his encouragements of the project, and Prof. T. G. Leighton (Southampton University) for his extensive comments on the manuscript.

\section{References}

Apfel, R. E. 1981 Acoustic cavitation prediction. J. Acoust. Soc. Am. 69, 1624-1633. (doi:10.1121/ $1.385939)$

Avellan, F., Henry, P. \& Rhyming, I. 1987 A new high-speed cavitation tunnel for cavitation studies in hydraulic machinery. In Proc. Int. Symp. on Cavitation Research Facilities and Techniques (FED 57) (eds J. W. Holl \& M. L. Billet), pp. 49-60. New York, NY: American Society of Mechanical Engineers.

Barber, B. P., Hiller, R. A., Löfstedt, R. \& Putterman, S. J. 1997 Defining the unknowns of sonoluminescence. Phys. Rep. 281, 65-144. (doi:10.1016/S0370-157|3(96)00050-6)

Ceccio, S. L. \& Brennen, C. E. 1991 Observations of the dynamics and acoustics of travelling bubble cavitation. J. Fluid Mech. 233, 633-660. (doi:10.1017/S0022112091000630) 
Couty, P., Farhat, M. \& Avellan, F. 2001 Physical investigation of cavitation vortex collapse. In Proc. 4th Int. Symp. on Cavitation (ed. C. E. Brennen), paper A6.003. Pasadena, CA: California Instutute of Technology.

Farhat, M., Pereira, F. \& Avellan, F. 1993 Cavitation erosion power as a scaling factor for cavitation erosion in hydraulic machines. In Proc. 3rd Int. Symp. on Cavitation Noise and Erosion (FED 176), pp. 95-104, New York, NY: American Society of Mechanical Engineering.

Frenzel, H. \& Schultes, H. 1934 Luminescenz im ultraschallbeschickten Wasser. Z. phys. chem. B 27, 421-424.

Guennoun, F., Farhat, M., Bouziad, Y. A. \& Avellan, F. 2003 Experimental investigation of a particular traveling bubble cavitation. Proc. 5th Int. Symp. on Cavitation (CAV2003), paper GS-2-012. Osaka, Japan: University of Osaka.

Harvey, E. N. 1939 Sonoluminescence and sonic chemiluminescence. J. Am. Chem. Soc. 61, 2392 2398. (doi:10.1021/ja01878a037)

Holland, C. K. \& Apfel, R. E. 1989 An improved theory for the prediction of microcavitation thresholds. IEEE Trans. Ultrasonics Ferroelectrics Frequency Control 36, 204-208. (doi:10.1109/58.19152)

Knapp, R. T. \& Hollander, A. 1948 Laboratory investigations of the mechanism of cavitation. Trans. Am. Soc. Mech. Engrs. 70, 419-435.

Leighton, T. G. 1994 The acoustic bubble. London, UK: Academic Press.

Leighton, T. G. 1998 Fundamentals of underwater acoustics and ultrasound. In Noise and vibration, vol. 1 (eds F. J. Fahy \& J. G. Walker), pp. 373-444. London, UK: E\&F Spon.

Leighton, T. G. 2007 What is ultrasound? Prog. Biophys. Mol. Biol. 93, 3-83. (doi:10.1016/ j.pbiomolbio.2006.07.026)

Leighton, T. G., Farhat, M., Field, J. E. \& Avellan, F. 2003 Cavitation luminescence from flow over a hydrofoil in a cavitation tunnel. J. Fluid Mech. 480, 43-60. (doi:10.1017/S0022112003003732)

Ohl, C.-D., Lindau, O. \& Lauterborn, W. 1998 Luminescence from spherically and aspherically collapsing laser induced bubbles. Phys. Rev. Lett. 80, 393-396. (doi:10.1103/PhysRevLett. 80.393)

Ohl, C.-D., Kurz, T., Geisler, R., Lindau, O. \& Lauterborn, W. 1999 Bubble dynamics, shock waves and sonoluminescence. Phil. Trans. R. Soc. Lond. A 357, 269-294. (doi:10.1098/rsta.1999.0327)

Peterson, F. B. \& Anderson, T. P. 1967 Light emission from hydrodynamic cavitation. Phys. Fluids 10, 874-879. (doi:10.1063/1.1762203)

Rayleigh, L. 1917 On the pressure developed in a liquid during the collapse of a spherical void. Philos. Mag. 34, 94-98.

van der Meulen, J. H. J. 1986 The relation between noise and luminescence from cavitation on a hydrofoil. In Proc. Joint ASCE/ASME Conf. on Cavitation in Hydraulic Structures and Turbomachinery (FED 25) (eds R. E. A. Arndt \& D. R. Webb), pp. 149-159. New York, NY: American Society of Mechanical Engineers.

van der Meulen, J. H. J. \& Nakashim, Y. 1983 A study of the relationship between type of cavitation erosion and luminescence. Proc. 2nd Int. Conf. on Cavitation, pp. 13-19. London, UK: Institution of Mechanical Engineers.

Weninger, K. R., Cho, H., Hiller, R. A., Putterman, S. J. \& Williams, G. A. 1997 Sonoluminescence from an isolated bubble on a solid surface. Phys. Rev. E 56, 6745-6749. (doi:10.1103/ PhysRevE.56.6745)

Weninger, K. R., Camara, C. G. \& Putterman, S. J. 1999 Energy focusing a converging fluid flow: implications for sonoluminescence. Phys. Rev. Lett. 83, 2081-2084. (doi:10.1103/ PhysRevLett.83.2081) 